\title{
Identification and Estimation of Harmonic Sources Based on Compressive Sensing
}

\author{
Daniele Carta, Student Member, IEEE, Carlo Muscas, Senior Member, IEEE, Paolo Attilio \\ Pegoraro, Member, IEEE, Sara Sulis, Member, IEEE
}

\begin{abstract}
Nodes in electric distribution networks are greatly differentiated and are very often nonlinear and/or unbalanced. They can create significant harmonic pollution, with harmonics that inevitably spread along the grid. Monitoring harmonic propagation and correlated power quality phenomena requires specific measurement devices and methodologies. Nevertheless, because of the unavailability of a rapid diffusion of synchronized and dedicated devices (due to technical and economic reasons) on every node and branch of the network, estimating the harmonic status of the entire grid by means of a complete or even redundant monitoring system can be practically unfeasible. A more feasible, though always meaningful, goal can thus be pursued, that is estimating the main harmonic sources in the network, rather than its complete harmonic status. This approach, of course, can be based on a simpler and cheaper upgrade of the distributed monitoring system. Even more, by considering the common scenario where the number of significant harmonic sources is lower than the number of loads connected to the grid, specific estimation procedures can be defined to further reduce the complexity of the monitoring system. In this scenario, this paper presents an efficient Compressive Sensing Harmonics Detector (CSHD) for the identification and the estimation of the principal pollution sources. The proposed CSHD method is validated by means of appropriate tests performed on an example of distribution grid.
\end{abstract}

Index Terms-Compressed sensing; Harmonic distortion; Harmonic Source Estimation; Matching pursuit algorithms; Power distribution

\section{INTRODUCTION}

In the last decades, distribution grids have been evolving rapidly and significantly towards highly complex systems, with an impressive growth of distributed generation and a large variety of loads. Most of them are power-electronics based, and this causes power quality (PQ) phenomena along the grid [1]. The PQ involves several scientific issues and practical consequences, with effects perceivable by final users. Poor PQ may cause damage and the total cost of electrical energy usage can be doubled. As an example, in 2015, the European Copper

D. Carta, C. Muscas, P. A. Pegoraro, S. Sulis are with the Department of Electrical and Electronic Engineering of the University of Cagliari, Piazza d'Armi, 09123 Cagliari, Italy (email: [daniele.carta, paolo.pegoraro, carlo, sara.sulis]@diee.unica.it). The work of Dr. Pegoraro was supported by the Fondazione di Sardegna for the research project SUM2GRIDS, Solutions by multidisciplinary approach for intelligent Monitoring and Management of power distribution GRIDS-CUP F71I17000290002.

(c) 2018 IEEE. Personal use of this material is permitted. Permission from IEEE must be obtained for all other users, including reprinting/republishing this material for advertising or promotional purposes, creating new collective works for resale or redistribution to servers or lists, or reuse of any copyrighted components of this work in other works. DOI:10.1109/TIM.2018.2838738 Publisher version:http://https://ieeexplore.ieee.org/document/8378234/
Institute estimated that the total PQ cost for European industry economy exceeds $150 \cdot 10^{9}$ euros [2].

In the industrial sector, the relevance of these phenomena is only partially perceived, because industrialists are typically more focused on other impairments, such as voltage interruptions. However, several studies draw the attention to the harmonic distortion [1], [3]. The harmonics reduce the life time of the components, incrementing costs, and their accurate identification is still an open challenge [4]-[6].

In this context, several papers presented in the literature have been focused on the evaluation of the harmonic pollution through the grid, applying Harmonic State Estimation methodologies (see for example [7]-[9]). These techniques, mostly derived from classic State Estimation methods originally designed for transmission systems, require a significant quantity of information, such as measured data, network topology and network parameters, to obtain an accurate evaluation of the quantities of interest. As a consequence, their implementation becomes impractical from an economic point of view.

Nevertheless, rather than knowing the overall harmonic state of the grid, it might be useful to identify correctly the harmonic pollution sources, to reduce/compensate their effects on the system, and, when possible, to attribute the costs arising from the disturbances. This can be a crucial aspect, above all when market liberalization impacts on electricity price.

In this scenario, specifically for the distribution networks, in [10] and [11] an approach called Harmonic Source Estimation was proposed. It consists in finding only the sources of harmonic pollution in the system and, therefore, requires less measurements than the evaluation of the whole harmonic state. It is worth noticing that the harmonic behavior of the distribution systems is usually poorly known, since still few real measurements are available on the field, commonly only in the high/medium voltage, HV/MV, stations, and very few PQ-meters are connected to the grid. Consequently, it is still not possible to monitor directly harmonic pollution in a large number of nodes of the grids. In this regard, an upgrade of the monitoring system is to be expected in the near future.

Nonetheless, at each moment, the number of significant harmonic sources in a distribution grid can be much lower than the total number of loads. This means that, practically, the model used for main harmonic sources identification can be considered as sparse. Based on this consideration, in this paper the problem of harmonic source identification and estimation is addressed by means of the Compressive Sensing (CS) theory, which is a signal recovery technique suitable to recover sufficiently sparse signals from limited 
information ([12], [13]). In particular, starting from the single harmonic identification approach presented in [14], where the main harmonic currents in the network were detected based on an Block Orthogonal Matching Pursuit (BOMP) algorithm, this paper introduces a new global Compressive Sensing Harmonics Detector (CSHD) that allows identifying the main polluting sources regardless of the specific harmonic involved, and estimating the corresponding injected harmonic currents.

The CSHD aims at giving an explorative tool, which can help the operator to investigate harmonic sources and decide how to carry on further and more targeted measurement campaigns. It is validated by means of appropriate simulation tests carried out on a small network derived from the 13bus IEEE network and practical aspects of the detection are discussed with reference to international standards. The proposed CSHD proves to be efficient in the identification of the principal polluting loads with respect to both the singleharmonic algorithm in [14] and an identification algorithm based on the classic WLS estimation.

\section{Proposed Harmonic Source IdEntification And ESTIMATION AlgORITHM}

\section{A. Definition of the Measurement Model}

The harmonic sources identification and estimation problem can be defined by means of the underlying measurement model in the frequency domain. The following general and linear model is adopted for each harmonic order $h$ :

$$
\overline{\mathbf{z}}_{h}=\overline{\mathbf{H}}_{h} \overline{\mathbf{u}}_{h}+\overline{\mathbf{e}}_{h}
$$

where $\overline{\mathbf{z}}_{h} \in \mathbb{C}^{M}$ includes $M$ phasor measurements (voltage or current measured phasors at harmonic $h), \overline{\mathbf{H}}_{h} \in \mathbb{C}^{M \times N}$ is the measurement matrix, $\overline{\mathbf{u}}_{h} \in \mathbb{C}^{N}$ is a vector whose entries are the $N$ unknown forcing terms (harmonic sources), and $\overline{\mathbf{e}}_{h}$ represents the phasor measurement errors vector. The over-bar represents complex quantities and all the involved quantities change depending on the harmonic order $h$. In detail, the measurement matrix $\overline{\mathbf{H}}_{h} \triangleq \overline{\mathbf{H}}\left(h f_{0}\right)$ represents the matrix frequency response of the network computed at frequency $h f_{0}$, with $f_{0}$ the fundamental frequency of the system. This matrix links the measured harmonic phasors to the input harmonic sources produced by the possible polluting loads or generators connected to the MV distribution system. $\overline{\mathbf{u}}_{h}$ includes all the sources of the $h$ th harmonic that can affect the network (usually currents injected by nonlinear loads or generators, but potentially also voltage supply) and impact on the measurements given by the PQ instrumentation located at different points of the network (included in $\overline{\mathbf{z}}_{h}$ ). For these reasons, the entries of $\overline{\mathbf{H}}_{h}$ can be computed from the impedances of lines and loads (see the network model in Section III-A, Fig. 1) and measurement relationships.

Equation (1) reflects the approach for harmonic measurements of international standards as [15], [16] and refers to steady-state or quasi-steady-state phenomena and measurements computed on sliding observation windows associated with given time tags.
The aim of the harmonic sources identification and estimation process is thus to find $\overline{\mathbf{u}}_{h}$ for each harmonic order $h$ of interest starting from the available phasor measurements $\overline{\mathbf{z}}_{h}$. A more suitable model to apply the algorithm keeping into account the accuracy of the measurements, is the following linear and real-valued model:

$$
\mathbf{z}_{h}=\left[\begin{array}{c}
z_{h, 1}^{r} \\
z_{h, 1}^{x} \\
\vdots \\
z_{h, M}^{r} \\
z_{h, M}^{x}
\end{array}\right]=\mathbf{A}_{h}\left[\begin{array}{c}
\mathbf{u}_{h}^{r} \\
\mathbf{u}_{h}^{x}
\end{array}\right]+\left[\begin{array}{c}
\mathbf{e}_{h}^{r} \\
\mathbf{e}_{h}^{x}
\end{array}\right]
$$

where $z_{h, i}^{r}$ and $z_{h, i}^{x}$ are the real and imaginary parts of the $i$ th measurement in $\overline{\mathbf{z}}_{h}$, respectively, and $u_{h}^{r}, u_{h}^{x}, e_{h}^{r}, e_{h}^{x}$ are similarly defined starting from the input and error vector. The measurement matrix $\mathbf{A}_{h}$ is defined as follows:

$$
\mathbf{A}_{h}=\left[\begin{array}{cc}
\mathbf{H}_{h}^{r} & -\mathbf{H}_{h}^{x} \\
\mathbf{H}_{h}^{x} & \mathbf{H}_{h}^{r}
\end{array}\right]
$$

where $\mathbf{H}_{h}^{r} \triangleq \Re\left[\overline{\mathbf{H}}_{h}\right]$ and $\mathbf{H}_{h}^{x} \triangleq \Im\left[\overline{\mathbf{H}}_{h}\right]$ (that is the real and imaginary parts of the complex measurement matrix).

Since a potentially polluting load or generator can be seen as a multiple harmonic producer, the model in (2) can be generalized into:

$$
\mathbf{z}=\left[\begin{array}{c}
\mathbf{z}_{h_{1}} \\
\vdots \\
\mathbf{z}_{h_{Q}}
\end{array}\right]=\left[\begin{array}{lll}
\mathbf{A}_{h_{1}} & & \\
& \ddots & \\
& & \mathbf{A}_{h_{Q}}
\end{array}\right]\left[\begin{array}{c}
\mathbf{u}_{h_{1}}^{r} \\
\mathbf{u}_{h_{1}}^{x} \\
\vdots \\
\mathbf{u}_{h_{Q}}^{r} \\
\mathbf{u}_{h_{Q}}^{x}
\end{array}\right]+\left[\begin{array}{c}
\mathbf{e}_{h_{1}}^{r} \\
\mathbf{e}_{h_{1}}^{x} \\
\vdots \\
\mathbf{e}_{h_{Q}}^{r} \\
\mathbf{e}_{h_{Q}}^{x_{2}}
\end{array}\right]
$$

where $Q$ denotes the total number of harmonics considered. In (4) the set of the measured phasors at different frequencies is expressed as a function of the forcing terms spectrum (for the considered harmonics). The overall measurement matrix is block-diagonal because of the orthogonality of the harmonics and the validity of the superposition principle. $\mathbf{e}$ is the overall measurement error vector.

While in [14] single harmonic detection was considered, in this paper the estimation of the injecting loads/generators is considered as direct estimation of $\mathbf{u} \triangleq$ $\left[\mathbf{u}_{h_{1}}^{r \mathrm{~T}}, \mathbf{u}_{h_{1}}^{x \mathrm{~T}}, \cdots, \mathbf{u}_{h_{Q}}^{r \mathrm{~T}}, \mathbf{u}_{h_{Q}}^{x \mathrm{~T}}\right]^{\mathrm{T}}$, where ' $\mathrm{T}$ ' indicates the trans-
pose.

\section{B. Identification and Estimation Algorithm}

In the following, the proposed algorithm for harmonic sources identification and estimation is presented. It is aimed at finding the loads that are main sources of harmonics and estimating the injected harmonic currents when significant. The forcing terms vector $\mathbf{u}$ can be assumed to be sparse. In fact, the number of main polluting loads/generators at a given time instant (corresponding to the time-tag of the measurements set) can be expected to be much smaller than the total number of loads. For this reason, each vector $\overline{\mathbf{u}}_{h}$ and, as a consequence, $\mathbf{u}$ can have a small number of significant entries with respect to its size, which correspond to the main injecting loads and to their non-negligible harmonic sources. 
In the following, a harmonic source will be usually represented as a harmonic current generator in parallel with the load impedance (or as a harmonic voltage generator with Thevenin representation) and thus assuming sparsity means considering only few significant generators to be active simultaneously.

In this context, the sources identification and estimation problem can be considered as a recovery problem under sparsity conditions and thus CS techniques can be exploited for its solution. In particular, considering the global harmonic model (4), the forcing vector $\mathbf{u}$ is considered to be blocksparse: the sources associated with each load/generator are considered as blocks of $\mathbf{u}$ that can be either zero or active (nonzero). Every available prior information on harmonic behavior of loads or plants can be considered, while defining the problem, or introduced in the model as additional constraints.

Grouping the forcing terms by source, the unknowns vector and the measurement matrix can be rearranged as:

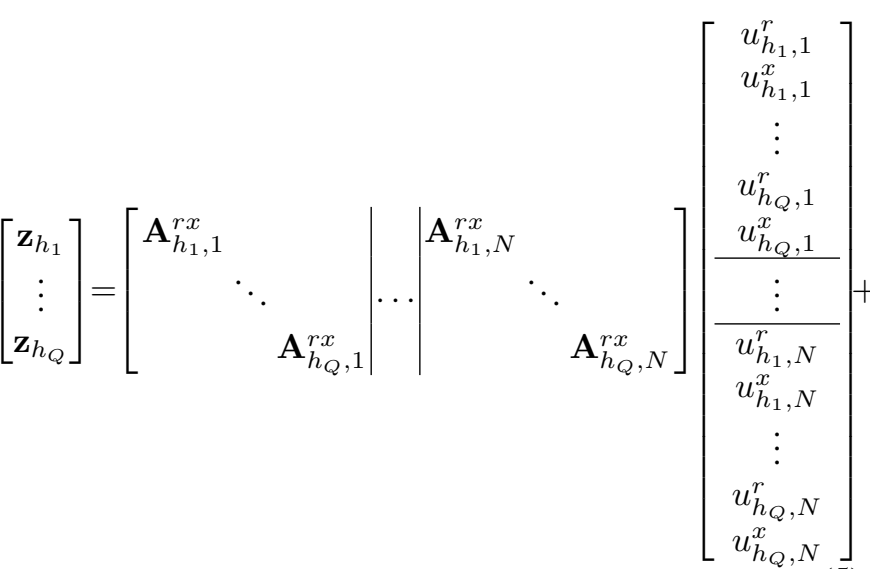

where $u_{k, l}^{r}(k=1, \ldots, Q$ and $l=1, \ldots, N)$ is the real part of the forcing quantity of the $l$ th possible injecting load/generator for the harmonic $k$ and an analogous definition holds for the imaginary part. $\mathbf{A}_{h_{k}, l}^{r x}=\left[\mathbf{a}_{h_{k}, l}^{r} \mathbf{a}_{h_{k}, l}^{x}\right]$ is the $M \times 2$ measurement matrix that corresponds to the $k$ th considered harmonic and the $l$ th source. In the following, the rearranged source vector in (5) is denoted as $\mathbf{s} \triangleq \mathbf{P u}$ ( $\mathbf{P}$ is the permutation matrix) and the rearranged overall measurement matrix is indicated by $\mathbf{L}=\mathbf{A} \mathbf{P}^{\mathrm{T}}$.

It is thus proposed to find a block-sparse solution for (5), by looking for a $\ell_{0}$-norm solution. The minimization problem can be generally defined, in the CS context, as follows:

$$
\hat{\mathbf{s}}=\underset{\mathbf{s}}{\arg \min }\|\mathbf{s}\|_{b 0} \text { s.t. }\|\mathbf{z}-\mathbf{L} \mathbf{s}\|_{2} \leq \epsilon
$$

where $\epsilon$ represents the bound of the noise energy [12] and $\|\mathbf{u}\|_{b 0}$ is the block- $\ell_{0}$-norm of $\mathbf{u}$, that is the number of nonzero source vectors $\mathbf{s}_{l} \triangleq\left[u_{h_{1}, l}^{r}, u_{h_{1}, l}^{x}, \cdots, u_{h_{Q}, l}^{r}, u_{h_{Q}, l}^{x}\right]^{\mathrm{T}}$ when $l=1, \cdots, N$.

For example, when the sparsity level is $S=2$, the solution becomes $\hat{\mathbf{s}}=\left[0, \cdots, 0, \mathbf{s}_{p 1}^{\mathrm{T}}, 0, \cdots, 0, \mathbf{s}_{p 2}^{\mathrm{T}}, 0, \cdots, 0\right]^{\mathrm{T}}$, corresponding to two injecting loads $p 1$ and $p 2$.

It has been demonstrated (see [17]) that (6) is NP-hard when recovering an unknown sparse signal. So, the solution is usually computed with approximated methods, and, in particular, with algorithms seeking the solution of a corresponding $\ell_{1}$-norm (or block- $\ell_{1}$-norm in the specific case) problem. In this way, the optimization problem becomes convex and, thus, easier to solve. Several algorithms have been proposed in the literature to face this problem [18]-[20]; in this paper a Block Orthogonal Matching Pursuit (BOMP) algorithm is adopted ([21], [22]). It is worth highlighting that while in [14] a BOMP algorithm was used at single-harmonic level, the new proposed algorithm is a BOMP algorithm performed at injecting/polluting element (hereinafter referred to as "load" for the sake of brevity) level. Since, in most cases, the harmonic sources are the loads, the new algorithm will be indicated as LBOMP in the following. A description of the procedure will be reported in Section II-D.

\section{Measurement Uncertainty}

The estimation framework represented by (5) and (6) has to be adapted to keep into account the measurement uncertainty. In the following, it is assumed that each harmonic phasor in $\mathbf{z}$ is measured with given accuracy. In particular, uncertainty in the measurement of magnitude and phase angle is considered. With most recent technologies, it is possible to imagine a monitoring scenario where synchronized phasor measurements for the harmonics of interest are available, e.g. from PMU-like devices with GPS receivers, as those presented and widely e installed in [23], [24], and operator efforts in this perspective are under way.

From suitable model of harmonic measurement uncertainty, it is thus possible to define, for each phasor, maximum deviations of magnitude and phase angle. Standard deviations of such measurements are computed by considering the underlying probabilistic distribution and a given level of confidence. In the following, uniform distributions are considered in the absence of other information.

Each measured phasor can be expressed in $\mathbf{z}$ in terms of its real and imaginary parts and the associated $2 \times 2$ covariance matrix is obtained by uncertainty propagation law [25]. The following expression is used:

$$
\begin{array}{r}
\boldsymbol{\Sigma}_{h_{k}, i}^{r x}=\left[\begin{array}{cc}
\cos \phi_{h_{k}, i} & -z_{h_{k}, i} \sin \phi_{h_{k}, i} \\
\sin \phi_{h_{k}, i} & z_{h_{k}, i} \cos \phi_{h_{k}, i}
\end{array}\right]\left[\begin{array}{cc}
\sigma_{z_{h_{k}, i}}^{2} & 0 \\
0 & \sigma_{\phi_{h_{k}, i}}^{2}
\end{array}\right] \\
{\left[\begin{array}{cc}
\cos \phi_{h_{k}, i} & \sin \phi_{h_{k}, i} \\
-z_{h_{k}, i} \sin \phi_{h_{k}, i} & z_{h_{k}, i} \cos \phi_{h_{k}, i}
\end{array}\right]}
\end{array}
$$

where $z_{h_{k}, i}=\left|\bar{z}_{h_{k}, i}\right|$ and $\phi_{h_{k}, i}$ are the magnitude and phase angle of the $i$ th measured phasor $\bar{z}_{h_{k}, i}=z_{h_{k}, i}^{r}+j z_{h_{k}, i}^{x}$ relating to the $k$ th considered harmonic.

In this paper all the measured phasors are considered independent and thus:

$$
\boldsymbol{\Sigma}_{\mathbf{z}}=\left[\begin{array}{ccc}
\boldsymbol{\Sigma}_{h_{1}, 1}^{r x} & & 0 \\
& \ddots & \\
0 & & \boldsymbol{\Sigma}_{h_{Q}, M}^{r x}
\end{array}\right]
$$

The analysis in the presence of correlations among harmonics and measurements is out of the scope of this paper, but it can be conceptually set into the same framework.

The model in (5) is thus changed as follows:

$$
\mathbf{z}^{\prime} \triangleq \mathbf{U}^{-\mathrm{T}} \mathbf{z}=\mathbf{U}^{-\mathrm{T}} \mathbf{L} \mathbf{s}+\mathbf{e}_{w}=\mathbf{L}^{\prime} \mathbf{s}+\mathbf{e}_{w}
$$


where $\mathbf{U}$ is obtained from the Cholesky factorization of $\boldsymbol{\Sigma}_{\mathbf{z}}$ (that is $\boldsymbol{\Sigma}_{\mathbf{z}}=\mathbf{U}^{-\mathrm{T}} \mathbf{U}$ ) and $\mathbf{U}^{-\mathrm{T}}$ denotes the transpose of its inverse, which can be computed since the measurement covariance matrix is positive-definite. This transformation is introduced to weight all the measurements according to their uncertainties and $\mathbf{e}_{w}$ is thus the result of measurement errors whitening.

\section{LBOMP procedure}

The LBOMP algorithm is then applied to the measurement vector $\mathbf{z}^{\prime}$ and is composed of the following stages:

1) Measurement matrix columns normalization. This is the first step of the algorithm and consists in the orthonormalization of the columns of $\mathbf{L}^{\prime}$ inside each column-block. The normalization is particularly important because it allows treating together different sources (currents or voltages) based on their effects. A new measurement matrix and a new source vector are thus obtained:

$$
\mathbf{z}^{\prime}=\mathbf{L}^{\prime} \mathbf{D} \mathbf{D}^{-1} \mathbf{s}+\mathbf{e}_{w}=\mathbf{L}_{n} \mathbf{s}_{n}+\mathbf{e}_{w}
$$

where $D$ is the orthonormalization matrix, $\mathbf{L}_{n}=\mathbf{L}^{\prime} \mathbf{D}$ and $\mathbf{s}_{n}=\mathbf{D}^{-1} \mathbf{s}$. If only normalization is applied, $D$ is diagonal and its element $d_{l, l}=\left\|L_{*, l}^{\prime}\right\|_{2}^{-1}$ (the inverse of the euclidean norm of the $l$ th column of $\mathbf{L}^{\prime}$ ).

2) Block OMP iterative algorithm. Similarly to [14], the estimation is obtained by a greedy procedure:

First, a vector of residuals is initialized to the measurements, that is $\mathbf{r}_{0}=\mathbf{z}^{\prime}$.

Then, the iteration process starts. At each iteration $i t>0$, the algorithm finds and selects the maximum energy inner product between the residual vector at previous iteration $\mathbf{r}_{i t-1}$ and the $2 Q$ columns of $\mathbf{L}_{n}$ relating to a given load (indicated as $\mathbf{L}_{n, l}$ ). Thus the selection of the source at iteration it can be written as:

$$
f_{i t}=\underset{l \in\{1, \cdots, N\} \backslash \Gamma_{i t-1}}{\arg \max }\left\|\mathbf{r}_{i t-1}^{\mathrm{T}} \mathbf{L}_{n, l}\right\|_{2}
$$

where $\Gamma_{i t-1}$ is the set of load indices already chosen during previous iterations.

Once the new load is chosen, the corresponding submatrix $\mathbf{L}_{n, f_{i t}}$ is added to the matrix that stores all the selected columns as follows:

$$
\mathbf{B}_{\Gamma_{i t}}=\left[\mathbf{B}_{\Gamma_{i t-1}} \mathbf{L}_{n, f_{i t}}\right]
$$

where $\mathbf{B}_{\Gamma_{i t-1}}$ indicates the submatrix of $\mathbf{L}_{n}$ composed of all the load submatrices selected during previous iterations. $\mathbf{B}_{\Gamma_{i t}}$ is thus of size $(M \cdot Q) \times(2 \cdot Q \cdot i t)$ and represents the measurement matrix for the newly defined model. Defining $\mathbf{s}_{n}\left(\Gamma_{i t}\right)$ as the subvector of $\mathbf{s}_{n}$ obtained by keeping all the elements corresponding to the column of $\mathbf{L}_{n}$ included in $\mathbf{L}_{n}\left(\Gamma_{i t}\right)$, the sparse solution at iteration it is obtained by the classic left-inverse computation as:

$$
\hat{\mathbf{s}}_{n}\left(\Gamma_{i t}\right)=\mathbf{B}_{\Gamma_{i t}}^{\dagger} \mathbf{z}^{\prime}=\left(\mathbf{B}_{\Gamma_{i t}}^{\mathrm{T}} \mathbf{B}_{\Gamma_{i t}}\right)^{-1} \mathbf{B}_{\Gamma_{i t}}^{\mathrm{T}} \mathbf{z}^{\prime}
$$

since $\mathbf{B}_{\Gamma_{i t}}$ is, in general, a rectangular tall matrix having full column rank.
The residuals vector is then updated as $\mathbf{r}_{i t}=\mathbf{z}^{\prime}-$ $B_{\Gamma_{i t}} \hat{\mathbf{s}}_{n}\left(\Gamma_{i t}\right)$ and the process continues with next iteration until the maximum number of iterations $S$ is reached.

3) Once the estimated sources vector $\hat{\mathbf{s}}_{n}$ is obtained, the actual forcing terms are computed by applying denormalization and thus $\hat{\mathbf{s}}=\mathbf{D} \hat{\mathbf{s}}_{n}$. The estimated vector allows to compute the complex values corresponding to the harmonic sources.

For the sake of comparison, in the Section III, the estimations performed with the proposed method are compared with those obtained with the single-harmonic estimator presented in [14] and with a WLS estimator defined using $\Sigma_{\mathbf{z}^{\prime}}^{-1}$ as weighting matrix and $\mathbf{L}^{\prime}$ as model matrix. Since the system can be underdetermined (typically $M \leq N$ ) this estimator (indicated as WLS in the following) computes the minimum Mahalanobis-norm (with respect to $\Sigma_{\mathbf{z}^{\prime}}$ ) solution of the system.

\section{E. Detection of Harmonic Sources}

The proposed algorithm selects at each iteration a candidate load to be labeled as an injecting and potentially polluting load and then refines the estimations of the forcing terms. This allows to directly consider as non-polluting all the loads that are not chosen during the process. Nevertheless, for a meaningful detection of the main sources it is also necessary to define post-estimation detection thresholds that prevent the algorithm from considering as polluting even loads that actually introduce negligible distortion. It is important to highlight that the algorithms looks for significant harmonic sources. Considerations on the harmonics propagation and on the actual polluting role of each injection at each time instant can be assessed only by targeted investigations, which can be triggered by the proposed identification.

The discussion on possible thresholds is presented in Section III, along with the detection results. Here it is important to highlight that the following rule is applied for each harmonic order $h_{k}$ and each load $l$ :

$$
v_{h_{k}, l}=\left(\sqrt{\hat{u}_{h_{k}, l}^{r 2}+\hat{u}_{h_{k}, l}^{x 2}} \geq t_{h_{k}, l}\right)=\left\{\begin{array}{l}
\text { true } \Rightarrow \text { polluting } \\
\text { false } \Rightarrow \text { non-polluting }
\end{array}\right.
$$

where $t_{h_{k}, l}$ is the threshold fixed for harmonic $h_{k}$ and load $l$, and must be tailored to the needs of the operator. It can generally depend on the type of the considered source, on the network characteristics and it can be written as a function of different parameters. In the following, it is considered as varying with the harmonic order and the nominal absorption characteristic of the load.

The rule in (14) is defined for each harmonic and thus it is also applied in Section III to other methods for the sake of fair comparison. Nevertheless it is important to underline that the proposed method labels as polluting a load $l$ when $\vee_{k=1}^{Q} v_{h_{k}, l}=$ true, which means that at least one harmonic generator for that load is above the chosen threshold. 


\section{TESTS AND RESULTS}

\section{A. Setup}

The system used to validate the proposed methodology is a single-phase distribution grid, derived from the IEEE 13 bus distribution test feeder [26]. The model of the grid, previously considered in the literature and already used also in [27], is presented in Fig. 1.

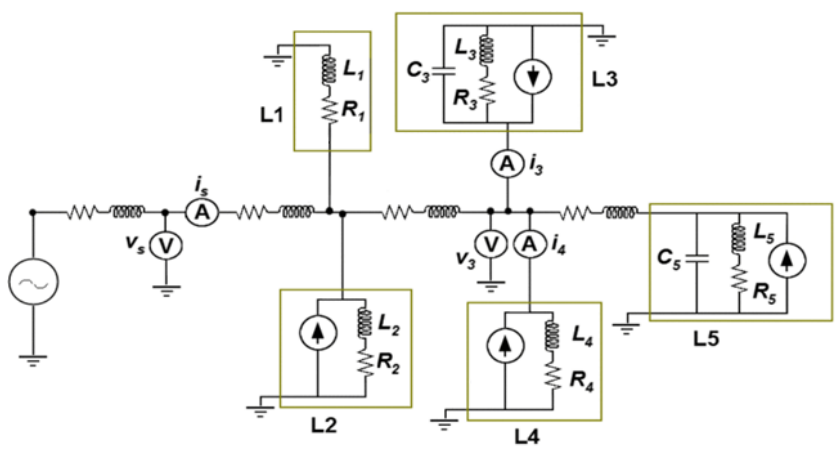

Fig. 1. Test grid.

The grid is composed by a voltage supply (nominal rms value $E_{s}=2,4 k V$ ) and five loads, characterized by the nominal active powers and corresponding nominal current, as reported in Table I. The measurement devices location is shown in Fig. 1. In particular, two devices, each with multiple measurement channels, are considered at two typical monitoring locations. No custom optimization of the device location has been performed. It is worth noticing that device technologies and architectures similar to those considered in [28] can be used for a in field implementation.

Each load is a clustered load, represented also in terms of an ohmic-inductive, RL, impedance. Loads clusters L3 and L5 also contain capacitors, connected in parallel with the RL impedance.

The non-linearity of the loads has been modeled by means of forcing harmonic current sources. These forcing sources have been placed in parallel with each load as harmonic current generators, except for load L1 that is always considered linear (see Fig. 1).

TABLE I

NOMinAL LOADS CONFIGURATION

\begin{tabular}{lcc}
\hline Load & $P_{n}[k W]$ & $I_{n}[A]$ \\
\hline L1 & 1333.33 & 55.51 \\
L2 & 80.15 & 39.00 \\
L3 & 73.90 & 34.46 \\
L4 & 345.33 & 180.47 \\
L5 & 140.72 & 65.07 \\
\hline
\end{tabular}

The forcing current of the $p$ th load $\left(i_{p}(t)\right)$ is obtained by summing its odd harmonic components $\left(i_{h_{k}, p}(t)\right)$, up to the 9-th $(h=1,3,5,7,9$ and $Q=5)$ order, as follows:

$$
\begin{gathered}
i_{p}(t)=\sum_{k=1}^{Q} i_{h_{k}, p}(t) \\
i_{h_{k}, p}(t)=\sqrt{2} I_{h_{k}, p} \sin \left(2 \pi h_{k} f_{0}+\phi_{h_{k}, p}\right)
\end{gathered}
$$

where $I_{h_{k}, p}$ and $\phi_{h_{k}, p}$ are, respectively, the rms value and the phase angle for the $p$ th load, referred to the harmonic order $h_{k}$. Default values for each harmonic source generator are reported in Table II, where the rms values are expressed in terms of percentage with respect to the fundamental component of the corresponding load $\left(I_{n}\right.$ in Table I). The settings of the tests permit enabling or disabling each forcing source, for single or multiple harmonic orders.

The reference values used in the tests have been evaluated by means of (15). Then, the uncertainties introduced by the measurement system have been taken into account by means of a Monte Carlo (MC) approach, based on 10000 simulations, in which additive terms are extracted from uniform distributions. In order to define the limit values of these distributions, standard IEC 61000-4-7 [15] has been considered. It refers to the accuracy requirements for both harmonic voltage and current measurements, by defining two different classes: Class I and Class II. In [15], the use of Class I instruments is recommended for accurate measurements, such as standard compliance and emission measurements, while Class II instruments are suggested for general purpose. In this paper, focused on the correct detection of possible polluting loads, Class I instruments have been considered. Class I uncertainties are reported in Table III, where $U_{m}$ and $I_{m}$ are the measured values of the voltage and current magnitudes for a generic harmonic, respectively, and $U_{n}$ and $I_{n}$ are the nominal voltage and current of the measurement device.

It is worth recalling that phase angle accuracies are not considered in [15]. In this paper the accuracy of the phase angle of the fundamental component is set to $1 \mathrm{crad}$, while the accuracy for the harmonic phase angle measurement is assumed to increase proportionally with the harmonic order.

The aforementioned accuracies are intended to represent the overall uncertainty, also including uncompensated transducer errors. However, if different uncertainty models are available, e.g. for instrument transformers, these models can be included in the procedure, without changing the core of the algorithm.

\section{B. Results}

In this section, the performance of the proposed method is presented and compared with other methods previously presented in the literature, in particular the method proposed in [14] and a WLS method briefly introduced in Section II.

In the following, main results will be reported in terms of the percentage of detection of the harmonic sources for each method and in terms of accuracy for the estimation of the harmonic current (or voltage) injected by those sources.

In this paper, the detection policy is based on a given detection threshold, relaying on the idea that the injection of a small harmonic current/voltage in the system can be negligible if its effects do not significantly affect the behavior of the grid. It is worth highlighting that the use of specific thresholds in the methodology can allow preventing false detections and neglecting the poorly polluting loads/generators, whereas a $0 \%$ threshold corresponds to consider as polluting a load whenever the corresponding algorithm output values for current/voltage magnitude are greater than zero. 
TABLE II

HARMONIC LOAD CONFIGURATION

\begin{tabular}{ccccccccc}
\hline \multirow{2}{*}{$\begin{array}{c}\text { harmonic } \\
\text { Order }\end{array}$} & \multicolumn{2}{c}{ Load L2 } & \multicolumn{2}{c}{ Load L3 } & \multicolumn{2}{c}{ Load L4 } & \multicolumn{2}{c}{ Load L5 } \\
\hline 3 & {$[\%]$} & $\phi_{h}[\mathrm{rad}]$ & $I_{h}[\%]$ & $\phi_{h}[\mathrm{rad}]$ & $I_{h}[\%]$ & $\phi_{h}[\mathrm{rad}]$ & $I_{h}[\%]$ & $\phi_{h}[\mathrm{rad}]$ \\
\hline 3 & 10.2 & -0.48 & 19.3 & -0.37 & 7.5 & -1.53 & 21.1 & -0.35 \\
5 & 2.1 & 0.89 & 3.8 & 1.12 & 3.7 & -0.33 & 4.1 & 1.16 \\
7 & 1.6 & 2.58 & 3 & 2.59 & 0.7 & 2.40 & 3.2 & 2.59 \\
9 & 1.0 & -1.67 & 1.9 & -1.67 & 0.5 & -1.69 & 2.1 & -1.67 \\
\hline
\end{tabular}

TABLE III

ACCURACY REQUIREMENTS FOR VOLTAGE AND CURRENT MEASUREMENTS [15]

\begin{tabular}{cccc}
\hline Class & Measurements & Conditions & Max Error \\
\hline \multirow{4}{*}{ I } & \multirow{2}{*}{ Voltage } & $U_{m} \geq 1 \% U_{n}$ & $\pm 5 \% U_{m}$ \\
& & $U_{m}<1 \% U_{n}$ & $\pm 0.05 \% U_{n}$ \\
\cline { 2 - 4 } & \multirow{2}{*}{ Current } & $I_{m} \geq 3 \% I_{n}$ & $\pm 5 \% I_{m}$ \\
& $I_{m}<3 \% I_{n}$ & $\pm 0.15 \% I_{n}$ \\
\hline
\end{tabular}

A realistic criterion to determine whether a harmonic component can be considered as really polluting or, on the contrary, negligible, can be based on information available in the technical report IEC/TR 61000-3-6 [16]. This report, which is informative in its nature, deals with distorting installations to MV, HV and extra-high-voltage (EHV) public power systems, considering as distorting an installation (which may be either a load or a generator) that produces harmonics and/or interharmonics. In particular, Table 2 in [16] defines indicative planning levels for harmonic voltages. These levels are equal or lower than the compatibility levels defined in Table 1 in [16], and their values depend on the system under study. As far as the loads currents are concerned, Table 5 in [16] defines, for some odd harmonic orders, indicative limits relative to the customer installations with an agreed power lower than or equal to 1 MVA.

In this paper, these relative harmonic current emission limits are considered as a starting point for the identification of the thresholds. For the sake of simplicity, the limits for the 3rd and 9th harmonic currents, which are not included in the mentioned Table 5 of [16], will be assumed to be, respectively, $5 \%$ (as for the 5 th and 7 th harmonics) and $3 \%$ of the nominal current (as for the 11th harmonic), as shown in Table IV.

TABLE IV

HARMONIC CURRENT EMISSION Limits From [16], EXTENDED With $3^{\text {RD }}$ AND $9^{\text {TH }}$ HARMONICS

\begin{tabular}{cccccccc}
\hline Harmonic order $\boldsymbol{h}$ & $\mathbf{3}$ & $\mathbf{5}$ & $\mathbf{7}$ & $\mathbf{9}$ & $\mathbf{1 1}$ & $\mathbf{1 3}$ & $>\mathbf{1 3}$ \\
Emission Limits [\%] & 5 & 5 & 5 & 3 & 3 & 3 & $500 / h^{2}$ \\
\hline
\end{tabular}

In real practice, such limits should be fixed depending on the operator needs and grid characteristics. For this reason, an analysis on possible effect of different values of detection thresholds has been performed and discussed.

In the following, the detection percentage will be plotted as a function of the defined threshold. In the plots, the values of [16], defined as percentages of the nominal current, will be reported in each figure with a red dotted line, labeled as "STD". Moreover, to better understand the behavior of each methodology, the actual value of the polluting load (as percentage of the nominal current) is indicated with a black dotted line, labeled as REF.

This type of graphical representation aims at testing the effects of different possible thresholds on the considered methodologies. The ideal detection curve (of a given harmonic due to an injecting load) can be represented by a step function located around the actual value of that harmonic (REF): the detection probability should be basically $100 \%$ for every threshold value below the REF value, after which it should fall quickly to $0 \%$. Therefore, when a load does not inject any harmonic current, the detection probability should be always zero, irrespective of the threshold level.

Among the high number of tests that have been performed, three different operating conditions are here discussed, to exemplify possible behaviors:

Case 1.

Forcing loads L3 and L4;

harmonic order under analysis $h=3$.

Case 2.

Forcing loads L4 and L5;

harmonic order under analysis $h=5$.

Case 3.

Forcing loads L3 and L4,

harmonic order under analysis $h=5$, under the hypothesis of load L3 producing harmonics without any 5 -th harmonic content.

The three considered detection methods are the BOMP proposed in [14] (blue line and circle marker in the figures), the WLS (red line and diamond marker), and the new proposed LBOMP methodology (yellow line and star marker).

Figure 2 shows the detection percentages as a function of the threshold level in Case 1, focusing on the results obtained for loads L3, L4 and L5. Both L3 and L4 loads are correctly detected by all the methods (100\%) whenever the detection threshold is lower than the REF.

Looking at the detection ratios corresponding to load L5, it is clear how the WLS algorithm can give a false detection, indicating L5 as a polluting load, while both BOMP and LBOMP detections are identical to the ideal curves (constantly equal to zero). The WLS curve reaches the $0 \%$ detection, in this specific case, with a threshold equal to $9 \%$ of the nominal current.

These results are also reported in Table $\mathrm{V}$, where the percentages of occurrence of detection are showed when the $5 \%$ threshold is applied, according to [16].

The mean values and the standard deviation of the absolute current magnitude estimation errors for Case 1 are reported in Table VI. Both BOMP and LBOMP algorithms give the same 

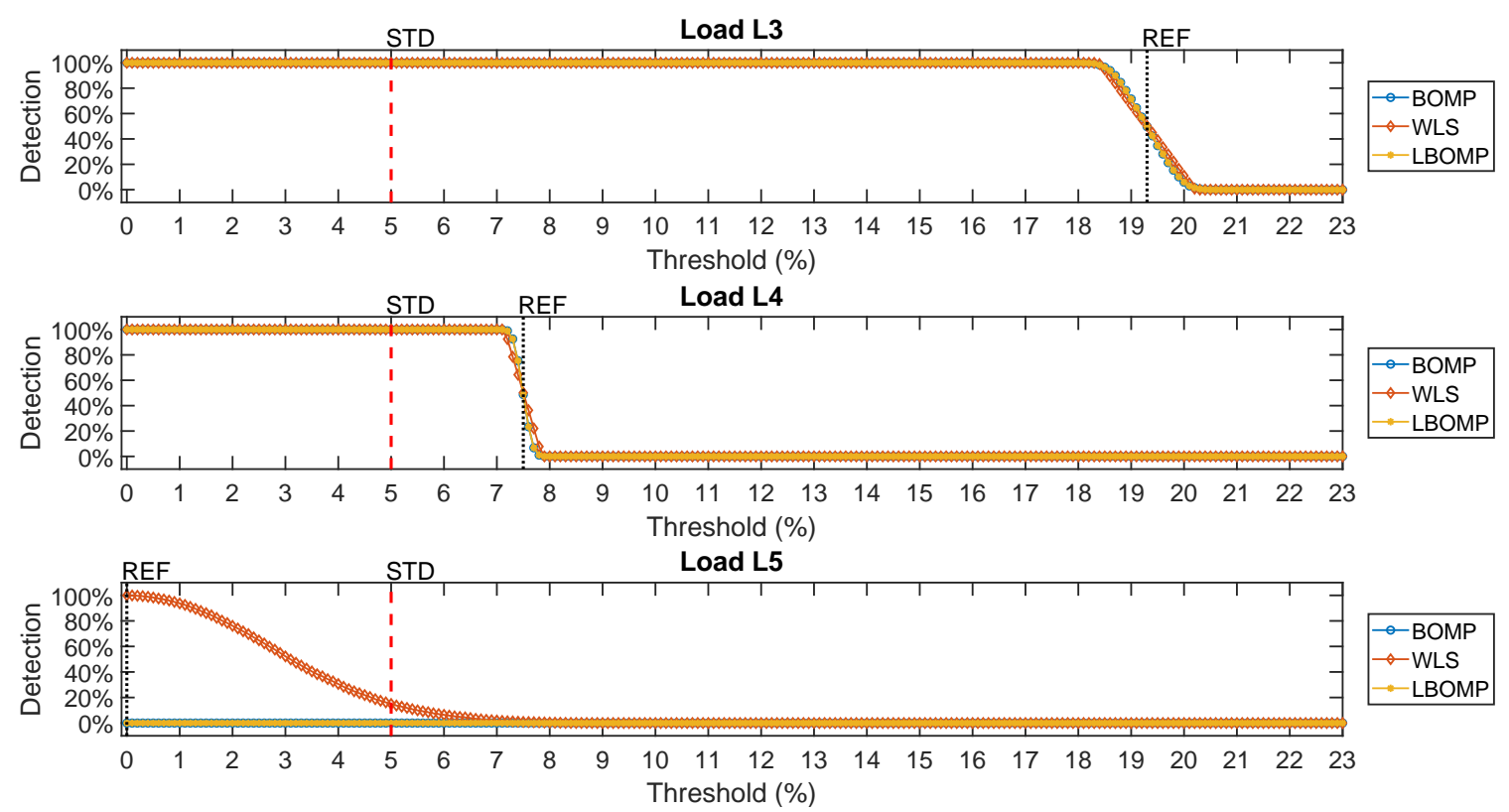

Fig. 2. Harmonic detection - Case 1: forcing loads L3 and L4, harmonic order under analysis $\mathrm{h}=3$.

TABLE V

Case 1, Percentage of Occurrence of Source Detection

\begin{tabular}{cccc}
\hline & BOMP & LBOMP & WLS \\
\hline L2 & 0 & 0 & 12.31 \\
L3 & 100 & 100 & 100 \\
L4 & 100 & 100 & 100 \\
L5 & 0 & 0 & 14.95 \\
\hline
\end{tabular}

results, while the WLS is characterized by a slightly higher error and standard deviation.

Similar considerations hold when all the loads not indicated as forcing in Case 1 actually inject harmonic currents, but with smaller amplitudes (5\% of Table II values have been tested). The same detection rates are found for LBOMP, while the estimation errors increase as expected (L3 and L4 mean absolute errors increase, respectively from $0.13 \mathrm{~A}$ to $0.22 \mathrm{~A}$ and from $0.2 \mathrm{~A}$ to $0.23 \mathrm{~A}$ ). In the following, all the tests are performed by setting at zero the harmonic currents injected by the loads that are not indicated as forcing in the three cases.

TABLE VI

Case 1, Means and Standard Deviations of Current Magnitude ESTIMATION AbSOLUTE ERRORS FOR LOADS L3, L4 AND L5

\begin{tabular}{ccccccc}
\hline & \multicolumn{2}{c}{ BOMP [A] } & \multicolumn{2}{c}{ LBOMP [A] } & \multicolumn{2}{c}{ WLS [A] } \\
& mean & std & mean & std & mean & std \\
\hline L3 & 0.13 & 0.16 & 0.13 & 0.16 & 0.16 & 0.18 \\
L4 & 0.20 & 0.24 & 0.20 & 0.24 & 0.32 & 0.37 \\
L5 & 0 & 0 & 0 & 0 & 2.12 & 1.05 \\
\hline
\end{tabular}

Figure 3 shows the results for Case 2. It is possible to see, for each considered algorithm, the correct detection of load L4. But, for the same case, the results for load L5 underline the differences among the methodologies under test. The LBOMP reaches $100 \%$ even for a threshold close to the REF value, while the BOMP detection percentage is lower (around $80 \%$ ) also for low thresholds. Even worst, WLS has a $100 \%$ detection rate only considering a zero threshold, while its capability of detection slowly decreases for increasing threshold values Furthermore, when the threshold is above REF, WLS still presents very large false detection rates. Moreover, in the threshold range between $1 \%$ and $9 \%$, the WLS curve corresponds to a high standard deviation of the estimation errors, as reported in the Table VII.

TABLE VII

Case 2, Means and Standard Deviations of Current Magnitude ESTIMATION ABSOLUTE ERRORS FOR LOADS L4 AND L5

\begin{tabular}{ccccccc}
\hline & \multicolumn{2}{c}{ BOMP [A] } & \multicolumn{2}{c}{ LBOMP [A] } & \multicolumn{2}{c}{ WLS [A] } \\
& mean & std & mean & std & mean & std \\
\hline L4 & 0.15 & 0.17 & 0.15 & 0.17 & 0.15 & 0.17 \\
$\mathbf{L 5}$ & 0.16 & 0.20 & 0.16 & 0.19 & 1.20 & 1.39 \\
\hline
\end{tabular}

Case 3 refers to the methodologies performance when load L3 injects at all harmonic orders, except for $h=5$. The main results are reported in Table VIII, focusing on the nonpolluting harmonic order. Two values for the threshold, namely the $5 \%$ defined in the IEC report [16] and one tenth of this value, i.e. $0.5 \%$, are considered to show the robustness of the proposed methodology also in this particular case.

TABLE VIII

Case 3, Percentage of Occurrence of Source Detection

\begin{tabular}{ccccccc}
\hline & \multicolumn{1}{c}{ BOMP } & \multicolumn{2}{c}{ LBOMP } & \multicolumn{2}{c}{ WLS } \\
& $0.5 \%$ & $5 \%$ & $0.5 \%$ & $5 \%$ & $0.5 \%$ & $5 \%$ \\
\hline L2 & 0 & 0 & 0 & 0 & 98.3 & 11.53 \\
L3 & 0 & 0 & 0 & 0 & 0.01 & 0 \\
L4 & 100 & 0 & 100 & 0 & 100 & 0 \\
L5 & 2.58 & 0 & 0 & 0 & 98.12 & 5.08 \\
\hline
\end{tabular}

The percentages of detection in Case 3 underline once again the validity of the proposed method. Looking at the results 

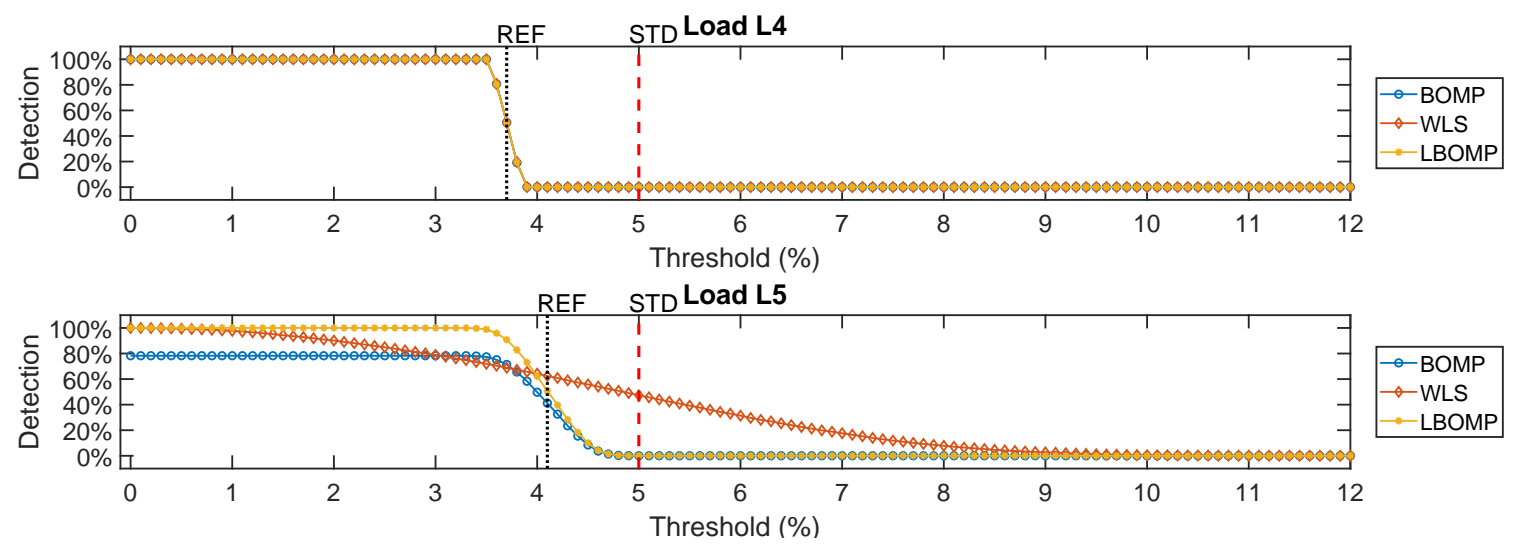

Fig. 3. Harmonic detection - Case 2: forcing loads L4 and L5, harmonic order under analysis $\mathrm{h}=5$.

obtained with a very low detection threshold, the LBOMP is the only method correctly detecting the real polluting load. The other methods obtain either a low false detection percentage (L5, BOMP) or false detections (L2 and L5, WLS). Meanwhile, the false detections are significantly reduced or eliminated by using the $5 \%$ threshold.

The mean and the standard deviation of the estimation errors in Table IX underline the better identification performances of the LBOMP with respect to the other algorithms. These results confirm that, in general, whereas both LBOMP and BOMP give good similar results in terms of estimation, the WLS algorithm gives a less trustworthy estimation which is characterized by higher errors and standard deviations.

TABLE IX

Case 3, Means and Standard Deviations of Current Magnitude ESTIMATION ABSOLUTE ERRORS FOR LOADS L3, AND L4

\begin{tabular}{ccccccc}
\hline & \multicolumn{2}{c}{ BOMP [A] } & \multicolumn{2}{c}{ LBOMP [A] } & \multicolumn{2}{c}{ WLS [A] } \\
& mean & std & mean & std & mean & std \\
\hline L3 & 0.08 & 0.03 & 0.06 & 0.03 & 0.07 & 0.04 \\
$\mathbf{L 4}$ & 0.12 & 0.15 & 0.09 & 0.11 & 0.15 & 0.17 \\
\hline
\end{tabular}

The reported results highlights the advantages of LBOMP. Then, focusing on the proposed algorithm, further tests based con Case 1 have been performed to demonstrate the effect of different measurement configurations.

The performance of LBOMP with only 4 measurements is considered removing $v_{3}$. The detection results with a $5 \%$ threshold are the same as before, with $100 \%$ of occurrence of source detection for both L3 and L4. However, as expected, the absence of $v_{3}$ affects the estimation of the forcing currents as showed in Table X. In particular the estimation errors increase of about $10 \%$ for L4 with respect to Table VI.

TABLE X

Case 1 without $v_{3}$, LBOMP Source Detection Rates, Means AND Standard DeViations of CURRENT Magnitude Estimation ABSOLUTE ERRORS FOR LOADS L3, L4

\begin{tabular}{cccc}
\hline & $\begin{array}{c}\text { Occurrence of Detection } \\
{[\%]}\end{array}$ & $\begin{array}{c}\text { mean } \\
{[\mathrm{A}]}\end{array}$ & $\begin{array}{c}\text { std } \\
{[\mathrm{A}]}\end{array}$ \\
\hline L3 & 100 & 0.13 & 0.16 \\
L4 & 100 & 0.22 & 0.27
\end{tabular}

In another test, considering the measurement placement of Fig. 1, the uncertainty of harmonic phase angle measurements is increased to a maximum deviation of $2 h$ crad, while keeping the maximum error for voltage and current magnitudes as in Table III. With this assumptions the same detection percentages are obtained, whereas the estimation errors of the found sources become up to $12 \%$ larger.

Finally, the robustness of the method is verified with respect to network model accuracy. In particular, the MC simulations have been also performed by including random variations (from uniform distributions with a maximum deviation of $\pm 20 \%$ ) in the load parameters. Also in this case, source detection is preserved, while the estimation results worsen (see Table XI). It is important to underline that, in this test, parameter uncertainty is not included in the model and thus reflects a pure model noise.

TABLE XI

Case 1 with Uncertain Load Parameters, Means and Standard DEVIATIONS OF CURRENT Magnitude Estimation Absolute ERrors FOR LOADS L3, L4

\begin{tabular}{cccc}
\hline & $\begin{array}{c}\text { Occurrence of Detection } \\
{[\%]}\end{array}$ & $\begin{array}{c}\text { mean } \\
{[\mathrm{A}]}\end{array}$ & $\begin{array}{c}\text { std } \\
{[\mathrm{A}]}\end{array}$ \\
\hline L3 & 100 & 0.18 & 0.22 \\
L4 & 100 & 0.43 & 0.53
\end{tabular}

\section{Conclusions}

This paper has presented an efficient and accurate Compressive Sensing Harmonics Detector, CSHD, for the identification and the estimation of the main harmonic sources in a power grid.

The proposed method relies on an appropriate definition of the measurement model and of the uncertainty contributions and it is based on a Block Orthogonal Matching Pursuit algorithm performed at the polluting source level.

The procedure permits identifying the main sources regardless of the specific harmonic involved, and estimating the corresponding harmonic currents injected in the grid. 


\section{APPENDIX}

\section{ORTHOGONALIZATION OF THE MEASUREMENT MATRIX}

In general, $\mathbf{D}$ in (10) is a block-diagonal matrix, since it includes all the orthogonalization bases for all the blocks of $\mathbf{L}^{\prime}$ relating to different loads. Denoting $\mathbf{L}_{l}^{\prime}$ as the $(2 \cdot M \cdot Q) \times(2 \cdot Q)$ submatrix including the columns associated with load $l$ and $\mathbf{L}_{l, h_{k}}=\left[\mathbf{l}_{l, h_{k}}^{r}, \mathbf{l}_{l, h_{k}}^{x}\right]$ the couple of $\mathbf{L}_{l}^{\prime}$ columns corresponding to the load forcing term for harmonic order $h_{k}, \mathbf{D} l$ th block is:

$$
\mathbf{D}_{l}=\left[\begin{array}{ccc}
\frac{1}{\left\|\mathbf{l}_{h_{1}, l}^{r}\right\|_{2}}-\frac{\mathbf{l}_{h_{1}, l}^{r} \cdot \mathbf{l}_{h_{1}, l}^{x}}{\left\|\mathbf{l}_{h_{1}, l}^{r}\right\|_{2}^{2}\left\|\mathbf{b}_{h_{1}, l}\right\|_{2}} & \\
0 & \frac{1}{\left\|\mathbf{b}_{h_{1}, l}\right\|_{2}} & \\
& & 1-\frac{\mathbf{l}_{h_{Q}, l}^{r} \cdot \mathbf{l}_{h_{Q}, l}^{x}}{\left\|\mathbf{l}_{h_{Q}, l}^{r}\right\|_{2}^{2}\left\|\mathbf{b}_{h_{Q}, l}\right\|_{2}} \\
& 0 & \frac{1}{\left\|\mathbf{b}_{h_{Q}, l}\right\|_{2}}
\end{array}\right]
$$

where $\left\|\mathbf{b}_{h_{1}, l}\right\|_{2}=\sqrt{\left\|\mathbf{l}_{h_{Q}, l}^{x}\right\|_{2}^{2}-\frac{\left(\mathbf{l}_{h_{Q}, l}^{r} \cdot \mathbf{l}_{h_{Q}, l}^{x}\right)^{2}}{\left\|\mathbf{l}_{h_{1}, l}^{r}\right\|_{2}^{2}}}$ and analogously for $\left\|\mathbf{b}_{h_{Q}, l}\right\|_{2}$. Matrix $\mathbf{D}_{l}$ is block-diagonal because of the orthogonality of the harmonics for each load.

\section{REFERENCES}

[1] M. H. J. Bollen, "What is power quality?" Electric Power Systems Research, vol. 66, no. 1, pp. 5-14, Jul. 2003. [Online]. Available: http://www.sciencedirect.com/science/article/pii/S0378779603000671

[2] European Copper Institute, Application Note, "The costs of poor Power Quality." [Online]. Available: http://www.leonardo-energy.org/ resources/297/the-cost-of-poor-power-quality-5800e490f1e14

[3] L. Cristaldi and A. Ferrero, "Harmonic power flow analysis for the measurement of the electric power quality," IEEE Transactions on Instrumentation and Measurement, vol. 44, no. 3, pp. 683-685, Jun. 1995.

[4] N. Locci, C. Muscas, and S. Sulis, "On the Measurement of PowerQuality Indexes for Harmonic Distortion in the Presence of Capacitors," IEEE Transactions on Instrumentation and Measurement, vol. 56, no. 5, pp. 1871-1876, Oct. 2007.

[5] A. Cataliotti, V. Cosentino, and S. Nuccio, "Comparison of Nonactive Powers for the Detection of Dominant Harmonic Sources in Power Systems," IEEE Transactions on Instrumentation and Measurement, vol. 57, no. 8, pp. 1554-1561, Aug. 2008.

[6] J. Barros, R. I. Diego, and M. d. Apraíz, "A Discussion of New Requirements for Measurement of Harmonic Distortion in Modern Power Supply Systems," IEEE Transactions on Instrumentation and Measurement, vol. 62, no. 8, pp. 2129-2139, Aug. 2013.

[7] K. K. C. Yu, N. R. Watson, and J. Arrillaga, "Error analysis in static harmonic State estimation: a statistical approach," IEEE Transactions on Power Delivery, vol. 20, no. 2, pp. 1045-1050, Apr. 2005.

[8] C. F. M. Almeida and N. Kagan, "Harmonic state estimation through optimal monitoring systems," IEEE Transactions on Smart Grid, vol. 4 , no. 1, pp. 467-478, Mar. 2013.

[9] C. Rakpenthai, S. Uatrongjit, N. R. Watson, and S. Premrudeepreechacharn, "On Harmonic State Estimation of Power System With Uncertain Network Parameters," IEEE Transactions on Power Systems, vol. 28, no. 4, pp. 4829-4838, Nov. 2013.

[10] G. D'Antona, C. Muscas, and S. Sulis, "Localization of Nonlinear Loads in Electric Systems Through Harmonic Source Estimation," IEEE Transactions on Instrumentation and Measurement, vol. 60, no. 10, pp. 3423-3430, Oct. 2011.

[11] G. D'Antona, C. Muscas, P. A. Pegoraro, and S. Sulis, "Harmonic Source Estimation in Distribution Systems," IEEE Transactions on Instrumentation and Measurement, vol. 60, no. 10, pp. 3351-3359, Oct. 2011.
[12] E. J. Candes and M. B. Wakin, "An Introduction To Compressive Sampling," IEEE Signal Processing Magazine, vol. 25, no. 2, pp. 21-30, Mar. 2008.

[13] M. Bertocco, G. Frigo, C. Narduzzi, C. Muscas, and P. A. Pegoraro, "Compressive Sensing of a Taylor-Fourier Multifrequency Model for Synchrophasor Estimation," IEEE Transactions on Instrumentation and Measurement, vol. 64, no. 12, pp. 3274-3283, Dec. 2015.

[14] D. Carta, C. Muscas, P. A. Pegoraro, and S. Sulis, "Harmonics Detector in Distribution Systems based on Compressive Sensing," in 2017 IEEE International Workshop on Applied Measurements for Power Systems (AMPS), Sep. 2017, pp. 1-5.

[15] Electromagnetic compatibility (EMC) - Part 4-7: Testing and measurement techniques - General guide on harmonics and interharmonics measurements and instrumentation, for power supply systems and equipment connected thereto, IEC 61000-4-7:2002+AMD1:2008 CSV, Oct. 2009.

[16] Electromagnetic compatibility (EMC) Part 3-6: Limits Assessment of emission limits for the connection of distorting installations to $M V, H V$ and EHV power systems, IEC TR 61000-3-6:2008, Feb. 2008.

[17] D. L. Donoho, "For most large underdetermined systems of linear equations the minimal 11-norm solution is also the sparsest solution," Communications on Pure and Applied Mathematics, vol. 59, no. 6, pp. 797-829, Jun. 2006. [Online]. Available: http://onlinelibrary.wiley.com/doi/10.1002/cpa.20132/abstract

[18] J. A. Tropp and A. C. Gilbert, "Signal Recovery From Random Measurements Via Orthogonal Matching Pursuit," IEEE Transactions on Information Theory, vol. 53, no. 12, pp. 4655-4666, Dec. 2007.

[19] V. M. Patel and R. Chellappa, "Compressive Sensing," in Sparse Representations and Compressive Sensing for Imaging and Vision, ser. SpringerBriefs in Electrical and Computer Engineering. Springer New York, 2013, pp. 315, dOI: 10.1007/978-1-4614-6381-8_2. [Online]. Available: http://link.springer.com/chapter/10.1007/978-1-4614-6381-8_2

[20] S. Chen, D. Donoho, and M. Saunders, "Atomic Decomposition by Basis Pursuit," SIAM Journal on Scientific Computing, vol. 20, no. 1, pp. 33-61, Jan. 1998. [Online]. Available: http://epubs.siam.org/doi/ abs/10.1137/S1064827596304010

[21] Y. Fu, H. Li, Q. Zhang, and J. Zou, "Block-sparse recovery via redundant block OMP," Signal Processing, vol. 97, pp. 162-171, Apr. 2014. [Online]. Available: http://www.sciencedirect.com/science/article/ pii/S0165168413004210

[22] Y. C. Eldar, P. Kuppinger, and H. Bolcskei, "Block-Sparse Signals: Uncertainty Relations and Efficient Recovery," IEEE Transactions on Signal Processing, vol. 58, no. 6, pp. 3042-3054, Jun. 2010.

[23] M. Pignati, M. Popovic, S. Barreto Andrade, R. Cherkaoui, D. Flores, J.-Y. Le Boudec, M. M. Maaz, M. Paolone, P. Romano, S. Sarri, T. T. Tesfay, D.-C. Tomozei, and L. Zanni, "Real-time state estimation of the EPFL-campus medium-voltage grid by using PMUs," in Innovative Smart Grid Technologies Conference (ISGT), 2015 IEEE Power Energy Society, 2015, pp. 1-5.

[24] A. von Meier, E. Stewart, A. McEachern, M. Andersen, and L. Mehrmanesh, "Precision micro-synchrophasors for distribution systems: A summary of applications," IEEE Transactions on Smart Grid, vol. 8, no. 6, pp. 2926-2936, Nov. 2017.

[25] JCGM, "Evaluation of data - guide to the expression of uncertainty in measurement," JCGM 100:2008, Sep. 2008.

[26] W. H. Kersting, "Radial distribution test feeders," IEEE Transactions on Power Systems, vol. 6, no. 3, pp. 975-985, Aug. 1991.

[27] C. Muscas, L. Peretto, S. Sulis, and R. Tinarelli, "Investigation on Multipoint Measurement Techniques for PQ Monitoring," IEEE Transactions on Instrumentation and Measurement, vol. 55, no. 5, pp. 1684-1690, Oct. 2006.

[28] P. A. Pegoraro, A. Meloni, L. Atzori, P. Castello, and S. Sulis, "Pmubased distribution system state estimation with adaptive accuracy exploiting local decision metrics and iot paradigm," IEEE Transactions on Instrumentation and Measurement, vol. 66, no. 4, pp. 704-714, Apr. 2017. 